\title{
Hemangioma subglótico tratado con propranolol. Una nueva alternativa terapéutica. Caso clínico
}

\author{
JAIME OSORIO M. ${ }^{1,2}$, PILAR GAJARDO O. ${ }^{2}$, CARLOS TAPIA M. ${ }^{1}$ \\ 1. Médico, Servicio de Otorrinolaringología. Clínica Indisa. \\ 2 Médico, Servicio de Otorrinolaringología. Hospital Barros Luco Trudeau.
}

\begin{abstract}
Propranolol for the treatment of subglotic hemangioma- A new therapeutic alternative

Infantile Subglottic Hemangiomas (ISH) are rare and potentially fatal. Many treatments have been described for this condition. Propranolol is a new therapeutic alternative, with successful results in skin and airway infantile hemangiomas. Case study: The case of an infant with ISH and $90 \%$ blockage of his airway is presented. The patient underwent a tracheostomy procedure and then treated with oral propranolol, showing a good response after a month of treatment, presenting $10 \%$ obstruction and consequently, ending the tracheostomy early. Conclusion: The discovery of the effect of propranolol in the ISH has revolutionized the management of this condition, displacing steroids and surgery as first-line treatments. This study discusses the current ISH management.
\end{abstract}

(Key words: Infant Hemangioma, airway, Propranolol).

Rev Chil Pediatr 2013; 84 (2): 182-188

\section{RESUMEN}

Los hemangiomas infantiles subglóticos (HIS) son poco frecuentes y potencialmente mortales. Muchos tratamientos se han descrito para los HIS. El propranolol es una nueva alternativa terapéutica, con resultados exitosos en hemangiomas infantiles de piel y de vía áerea. Caso clínico: Se reporta el manejo de un lactante con HIS que presentaba una obstrucción del $90 \%$ de su vía aérea, que debió ser traqueostomizado, luego se inicio propranolol oral, presentando una muy buena respuesta al mes de tratamiento, con $10 \%$ de obstrucción, retirando traqueostomía precozmente. Conclusión: El descubrimiento del efecto del propranolol en los HIS ha revolucionado su manejo, desplazando a los corticoides y a la cirugía como primera línea de tratamiento. En el presente reporte se discute el manejo actual de los HIS.

(Palabras clave: Hemangioma infantil, vía aérea, Propranolol).

Rev Chil Pediatr 2013; 84 (2): 182-188

Recibido el 18 de junio de 2012, devuelto para corregir el 13 de agosto de 2012, segunda versión el 3 de octubre de 2012, aceptado para publicación el 5 de diciembre de 2012.

Este trabajo cumple con los requisitos sobre consentimiento /asentimiento informado, comité de ética, financiamiento, estudios animales y sobre la ausencia de conflictos de intereses según corresponda.

Correspondencia a:

Jaime Osorio M.

E-mail: jaime_osorio@yahoo.com 


\section{Introducción}

Los hemangiomas infantiles son lesiones vasculares caracterizadas por un rápido crecimiento durante los primeros meses de vida. Histológicamente, se caracterizan durante su fase de proliferación por un alto índice mitótico de células endoteliales dentro de los capilares, seguido por apoptosis y la sustitución con tejido fibroadiposo a medida que progresa a la fase de involución. Todos los hemangiomas infantiles presentan inmunorreactividad histológica positiva frente al GLUT-1 que corresponde a un transportador de glucosa transmembrana que se expresa en las células endoteliales de hemangiomas infantiles, placenta y barrera hematoencefalica ${ }^{1-3}$ y no en otros endotelios, ni otros tumores vasculares.

Estas lesiones benignas son los tumores más frecuentes en la infancia, con una incidencia entre un $5 \%$ a $15 \%$ en caucásicos. Es más frecuente en mujeres, prematuros y recién nacidos menores de $1.500 \mathrm{~g}$. E1 80\% de los hemangiomas se localizan en cabeza y cuello ${ }^{1-3}$.

Los hemangiomas infantiles requieren tratamiento cuando presentan riesgo vital, presentan alteraciones funcionales tales como compromiso de vía área y cuando presentan deformaciones cosméticas de importancia ${ }^{1-3}$.

El hemangioma infantil subglótico (HIS) es una condición rara que puede ser potencialmente mortal. Fue descrito por primera vez por Morrell Mackenzie en 1864 y representa aproximadamente el 1,5\% de todas las anomalías congénitas laríngeas ${ }^{4}$.

Las primeras semanas de vida suelen ser asintomáticos, ya que la fase de proliferación comienza después del primer mes de vida. Clínicamente se presentan como estridor inspiratorio o bifásico, dificultad respiratoria y disfagia, generalmente no presentan disfonía.

El diagnóstico preciso se realiza mediante la visualización endoscópica de la vía aérea. Se presenta como una lesión ubicada en la subglotis, de localización submucosa, de color rosada o purpura que a la palpación es suave, asimétrica y compresible. No se recomienda la realización de biopsia para el diagnóstico, debido a su alto riesgo de sangrado.

La historia natural de los hemangiomas infantiles es de un crecimiento importante durante los primeros meses de vida y luego una involución lenta y espontánea a partir de los doce meses de vida. Se describe una resolución completa del 50\% a los cinco años, 70\% a los siete años y el 90\% alrededor de los diez años de vida ${ }^{4}$.

El objetivo del tratamiento es mantener la vía aérea permeable los primeros años de vida, acortar el curso natural de la lesión y reducir los efectos secundario de la terapia. Numerosas modalidades se han utilizado para el tratamiento de HIS, dentro de los cuales se encuentran los corticoides sistémicos, corticoides intralesionales, interferón alfa, cirugía, y descrito recientemente, el propranolol ${ }^{4}$.

En el año 2008 Leaute-Labreze describió el efecto beneficioso del propranolol en el tratamiento de hemangiomas infantiles en piel, lo cual ha generado una revolución para el abordaje de este tipo de lesiones ${ }^{5}$.

Denoyelle y Buckmuller en forma separada, en el año 2009, reportaron el uso de propanol en HIS con resultados satisfactorios ${ }^{6,7}$.

\section{Objetivo}

El objetivo de este trabajo es reportar un caso de HIS tratado con propranolol y discutir su manejo.

\section{Caso clínico}

Solicitan evaluación Otorrinolaringológica en la Unidad de Tratamiento Intensivo (UTI) pediátrica de Clínica Indisa, por lactante de sexo femenino de un mes dieciséis días de vida, sin antecedentes mórbidos de importancia.

El motivo de la evaluación es un segundo episodio de laringitis con estridor congénito bifásico de predominio inspiratorio que aumenta con el llanto. La paciente había sido ingresada el día anterior a esa unidad por dificultad respiratoria caracterizada por estridor inspiratorio, retracción intercostal y tiraje, que respondió a nebulización con adrenalina y corticoides. La paciente se encontraba en buen estado general, afebril y un panel viral realizado al ingreso fue negativo.

Al examen físico destaca malformación 
vascular capilar en zona occipital, conocido como nevo de unna, sin lesiones vasculares cutáneas en la región mentoniana. Se realiza nasofibroscopía flexible que muestra una lesión subglótica de coloración rojiza en región posterolateral izquierda. Se explica el diagnóstico a la familia, pero como evoluciona satisfactoriamente mejorando su mecánica respiratoria, sólo manteniendo su estridor basal, familiares solicitan el alta. Reingresa a las dos semanas con estridor, disfonía, dificultad respiratoria y rechazo alimentario.

Se realiza tomografía computada (TC) de cuello que muestra disminución del calibre de la vía aérea a nivel subglótico en relación a lesión vascular de $5,1 \mathrm{~mm}$ de diámetro transverso, 5,5 $\mathrm{mm}$ de diámetro anteroposterior y 8,1 $\mathrm{mm}$ de longitud, compatible con el diagnóstico de hemangioma subglótico (figuras 1 y 2).

Se realiza laringoscopía directa en pabellón con óptica rígida de $0^{\circ}$ grados que muestra una lesión submucosa en región subglótica de base posterolateral izquierda, de color rojiza, compresible, que ocupa el 90\% del lumen de la vía área, por lo que se decide realizar traqueostomía, previa firma del consentimiento informado por los padres de la paciente (figura 3 ).

Se realizó evaluación cardiológica con ecocardiograma, electrocardiograma y exámenes generales, no habiendo contraindicación para el uso de propranolol oral en la paciente.

Se envió a preparar a farmacia comercial jarabe de propranolol con una concentración de $2 \mathrm{mg}$ por un $\mathrm{ml}$.

Se completaron diez días de tratamiento con corticoides orales y conjuntamente se inicio propranolol oral en dosis de $0,5 \mathrm{mg} / \mathrm{kg} /$ día hasta llegar $2 \mathrm{mg} / \mathrm{kg} /$ día en cuarenta y ocho horas, fraccionado en tres dosis. Se evaluó frecuencia cardíaca, presión arterial y niveles de glicemia durante todo el tratamiento. El tratamiento con propranolol fue bien tolerado por la lactante, sin efectos adversos a la terapia con este beta bloqueador.

Se reevalúa en pabellón a los treinta días de iniciado el tratamiento con propranolol, mostrando una importante remisión del hemangioma, quedando $10 \%$ de obstrucción de vía aérea (figura 4). Se retira cánula de traqueostomía al día siguiente. Se mantiene hospitali- zada por siete días en UTI en donde se retira sonda nasoenteral, se realimenta por vía oral, con video deglución normal y sin estridor, por lo que se da de alta. El cierre espontaneó de la traqueotomía se logra a las dos semanas del retiro de la cánula.

En el control a los cuatro meses de tratamiento con propranolol oral la paciente está sin estridor, ni dificultad respiratoria, con buen incremento del peso y no ha presentado complicaciones por el uso de propranolol. Mediante laringoscopía directa en pabellón, se observa una obstrucción del $30 \%$ de la vía aérea (figura 5).

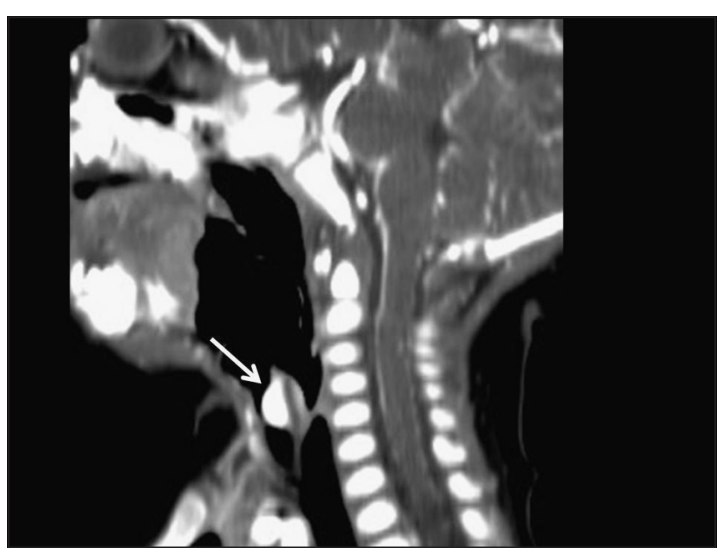

Figura 1. Tomografía computada de cuello con contraste endovenoso, reconstrucción sagital. Se demuestra lesión que se refuerza intensamente en la región subglótica (flecha).

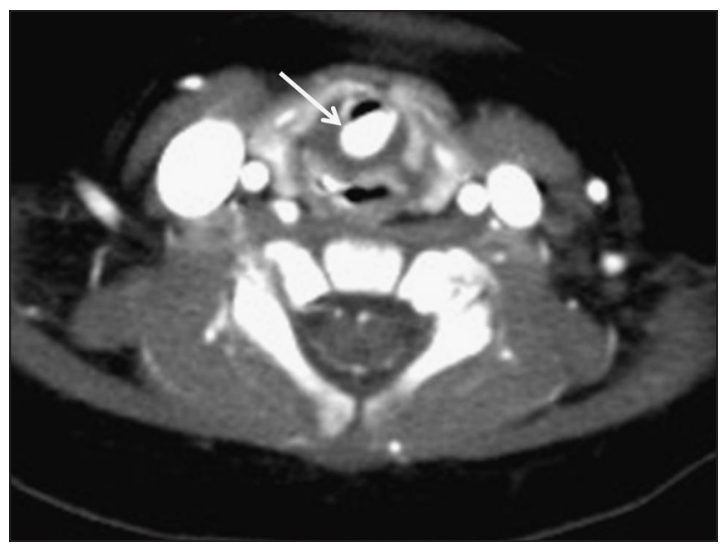

Figura 2. Tomografía computada de cuello con contraste endovenoso, corte axial. Se demuestra la lesión que se refuerza intensamente en la región subglótica y determina importante disminución del calibre de la vía aérea a ese nivel (flecha). 


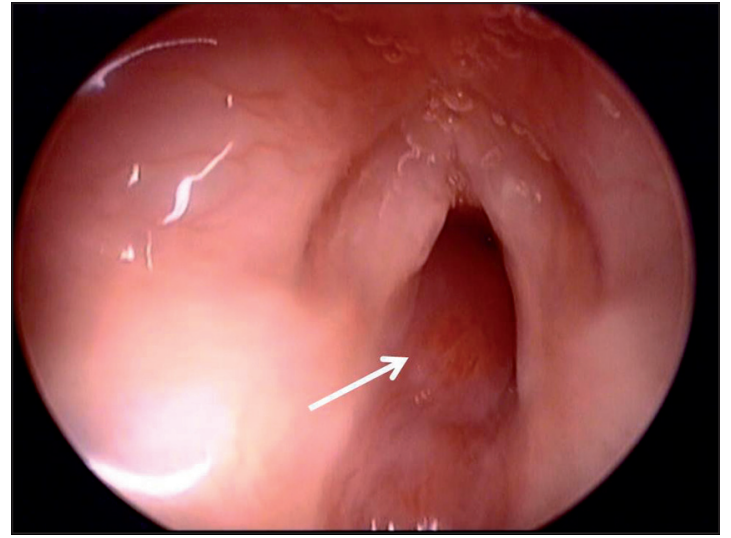

Figura 3. Laringoscopía directa con óptica de $0^{\circ}$ que muestra obstrucción de la vía aérea por el hemangioma, estimada en $90 \%$ (flecha).

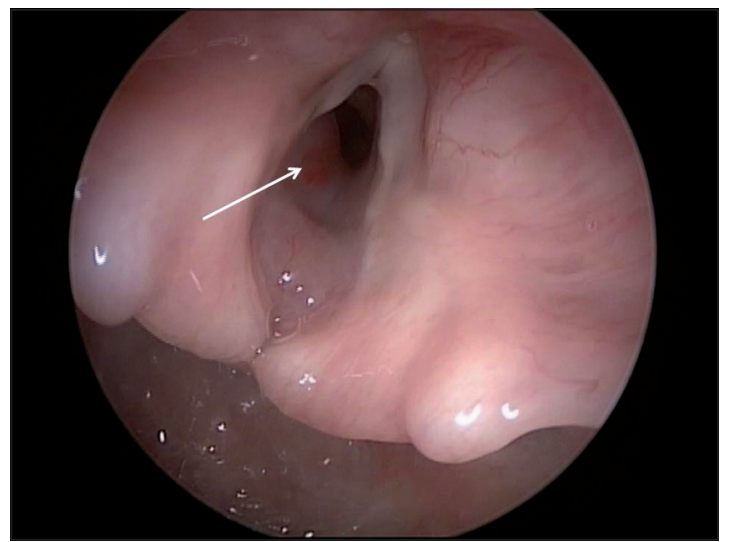

Figura 5. Laringoscopía directa con óptica de $0^{\circ}$ de control. La obstrucción se estima en $30 \%$, asintomática.

Hasta el momento de la publicación la paciente ha evolucionado asintomática con buen incremento de peso corporal y sin necesidad de nuevas hospitalizaciones por dificultad respiratoria.

\section{Discusión}

La traqueostomía como único manejo de HIS, para esperar y ver la evolución natural de esta patología benigna de vía aérea, logra el retiro de la cánula, en promedio, a los diecisiete meses de edad, pero presenta variadas complicaciones, tales como una mortalidad de $1-2 \%$, retraso del lenguaje, infecciones respiratorias

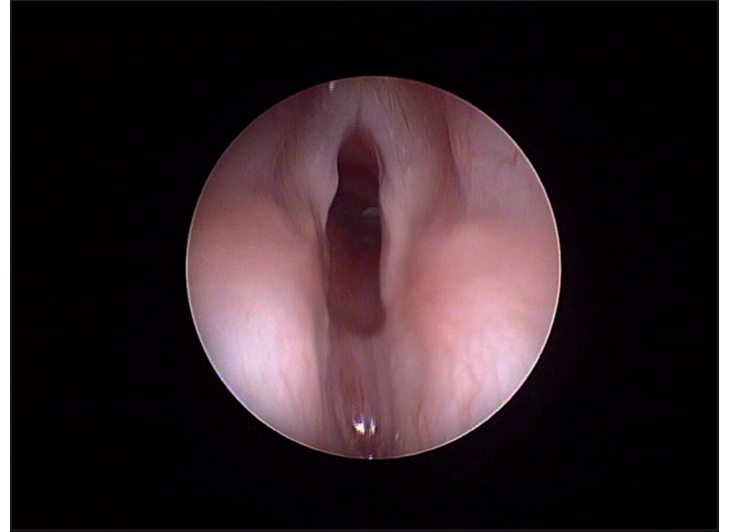

Figura 4. Control al mes de tratamiento con propanolol. Laringoscopía directa con óptica de $0^{\circ}$ muestra redución del volumen del hemangioma.

y alteración de la vida diaria para el paciente y su familia ${ }^{8}$.

Antes de la llegada del propranolol, los corticoides sistémicos eran el tratamiento de primera línea para los HIS, con una tasa de respuesta del $25 \%$. Sin embargo, aquellos pacientes que respondían debían permanecer con altas dosis de corticoides ( $3 \mathrm{mg} / \mathrm{kg} /$ día $)$ por tiempos prolongados con los efectos colaterales propios de la corticoterapia prolongada. Existen reportes de infiltraciones intralesionales de corticoides con una tasa de éxito de $83 \%$, pero esta técnica no ha sido adoptada globalmente, ya que los pacientes deben permanecer intubados por un tiempo promedio de cincuenta días y porque se requieren múltiples aplicaciones, lo cual eleva el costo del tratamiento?.

El interferón alfa $2 b$ y la vincristina se han utilizado con buena respuesta, pero con múltiples efectos colaterales, por lo que se consideran opciones de segunda o tercera línea en el enfrentamiento terapéutico de los HIS ${ }^{9,10}$.

Dentro de las alternativas quirúrgicas, la resección endoscópica con Láser de $\mathrm{CO}_{2}$ presenta una tasa global de éxito de $89 \%$. Esta técnica requiere múltiples procedimientos con una media de dos (intervalo de una a cinco intervenciones). Es importante señalar que existe el riesgo de desarrollar estenosis subglótica con este tipo de resección, la cual ha sido reportada en hasta un $25 \%$ de los pacientes sometidos 
a esta técnica quirúrgica, presentando mayor riesgo las lesiones circunferenciales ${ }^{10}$.

En algunos centros otorrinolaringológicos pediátricos se manejan los HIS obstructivos con resección por vía abierta y reconstrucción laringotraqueal, evitando la traqueostomía o siendo ésta transitoria. Se reporta un éxito entre un $82-96 \%$ de los casos, en un acto quirúrgico. Actualmente se usa para lesiones que no responden a tratamiento con corticoides sistémicos o propranolol. Sus desventajas son que requiere la hospitalización en unidades de cuidados intensivos y cirujanos entrenados ${ }^{11,12}$.

Nadie imagino que un hallazgo incidental el año 2008 iba a cambiar el manejo de los hemangiomas infantiles y en especial los HIS, pudiendo manejar estas lesiones con propranolol oral ${ }^{13,14}$.

El propranolol tiene las siguientes ventajas: 1. No es un tratamiento invasivo; 2. Presenta una rápida respuesta; 3. Evita, eventualmente, la traqueotomía; 4. Evita el tratamiento prolongado con corticoides y sus efectos adversos; 5. Evita la manipulación subglótica; 6. Evita los períodos prolongados de intubación; 7. Tiene una baja tasa de complicaciones y 8 . Tiene un bajo costo ${ }^{16}$.

El propranolol es un antagonista beta adrenérgico no selectivo, que inhibe competitivamente los receptores B1 y B2 adrenérgicos y con la misma afinidad que los ligandos naturales de estos receptores. El propranolol es un antagonista puro sin efectos agonistas parciales ${ }^{17}$.

El mecanismo de acción del propranolol aún no se conoce del todo, pero se postula que su acción se debe a 1 . Vasoconstricción, secundaria a la disminución de la síntesis de óxido nítrico; 2 . Inhibición de la angiogénesis por disminución de factores proangiogénicos y 3. Inducción de la apoptosis por activación de las caspasas ${ }^{17}$.

Los efectos adversos potenciales del propranolol son bradicardia, hipotensión, broncoespasmo, hipoglicemia, insomnio, agitación, sudoración nocturna y manos frías. Se describe que entre 3 a $18 \%$ de los pacientes sometidos a tratamiento con propranolol presentan efectos adversos a esta terapia, siendo mejor tolerado que los corticoides sistémicos ${ }^{18-20}$.
Los protocolos actuales para el uso de propranolol sugieren previo al inicio del tratamiento, evaluación cardiológica con signos vitales, ecocardiografía, electrocardiograma, glicemia basal, perfil bioquímico y perfil tiroideo. El inicio de propranolol se puede realizar con hospitalización abreviada de cuarenta y ocho horas o visitas domiciliarias de enfermería. Se debe controlar de forma seriada la presión arterial y la glicemia al inicio del tratamiento ${ }^{18-20}$.

Se recomienda una dosis inicial de 0,16 $\mathrm{mg} / \mathrm{kg} / \mathrm{dosis}$ cada $8 \mathrm{~h}$ y de forma incremental doblando a una dosis final de $0,67 \mathrm{mg} / \mathrm{kg} /$ dosis (equivalente a $2 \mathrm{mg} / \mathrm{kg} /$ día) en cuarenta y ocho horas $^{15}$.

El propranolol está contraindicado en pacientes con asma bronquial, shock cardiogénico, bloqueo cardíaco de segundo y tercer grado, hipersensibilidad al propranolol y bradicardia sinusal ${ }^{15}$.

La evidencia del uso de propranolol en HIS en la literatura se limita a casos clínicos, series de casos y estudios observacionales.

Leboulanger publicó la experiencia francesa en 2010 con doce casos en forma retrospectiva con muy buena respuesta al propranolol, al igual que otras series más pequeñas ${ }^{15}$.

Perides en su meta análisis muestra que el propranolol es el mejor tratamiento disponible actualmente para el tratamiento de HIS, siendo seguro y eficaz.

En la actualidad están en desarrollo diez estudios controlados para evaluar la eficacia y seguridad del propranolol y otros beta bloqueadores en hemangiomas infantiles, lo cual le dará mayor validez al uso de esta alternativa terapéutica en HIS (www.ClinicalTrials. gov) ${ }^{19}$.

A pesar de lo exitosos de los casos clínicos reportados, se describen fallas al tratamiento con propranolol en HIS, con una buena respuesta inicial y luego rebote a pesar del aumento dosis, por lo que hay que controlar estrechamente a estos pacientes ante la aparición de síntomas. Se aconseja realizar laringoscopía mensualmente para evaluar la vía área en estos pacientes ${ }^{21-24}$.

Fuchsmann reporta seis casos de recurrencia posterior a la suspensión del tratamiento en treinta $\mathrm{y}$ dos pacientes con hemangioma 
infantil en cabeza y cuello con un promedio de tratamiento con propranolol de ocho meses (rango entre siete a once meses), respondiendo todos al reinicio del tratamiento con propranolol. Esto se debería al componente profundo del hemangioma, al retiro precoz del medicamento o prolongación de la fase proliferativa de esta lesión benigna ${ }^{25}$.

Aún no existe consenso en cuanto tiempo debe durar el tratamiento, lo lógico sería mantenerlo durante la fase proliferativa de HIS, es decir, durante el primer año de vida, aunque se han reportado casos de duración del tratamiento con este medicamento, hasta los dos años de edad.

En el caso de nuestra paciente, la lactante debió ser sometida a una traqueostomía transitoria por la clínica de la paciente y lo obstructivo del hemangioma en la evaluación inicial (90\%). Por la buena respuesta al tratamiento con propranolol se pudo retirar la traqueostomía al mes sin complicaciones. Si bien en su último control se observó un aumento de volumen del HIS, completamente asintomático, éste debe ser seguido en forma estrecha debido a la posibilidad de un rebote.

\section{Conclusión}

Se describe el manejo de un HIS con propranolol, una nueva opción terapéutica y una alternativa de primera línea. Al igual que en la literatura internacional, el propranolol es eficiente en reducir los síntomas y el tamaño del HIS de nuestra paciente, presentando una rápida respuesta, sin efectos adversos.

Cada vez existen menos dudas y más evidencia de la utilidad de propranolol en los HIS. Estudios complementarios de mejor calidad son necesarios para comprender el verdadero papel del propranolol en la fisiopatología y el tratamiento de los hemangiomas infantiles.

\section{Referencias}

1.- Werner J, Dünne A, Folz B, et al: Current concepts in the classification, diagnosis and treatment of hemangiomas and vascular malformations of the head and neck. Eur Arch Otorhinolaryngol 2001; 258: 141-9.
2.- Puttgen K, Pearl M, Tekes A, et al: Update on pediatric extracranial vascular anomalies of the head and neck. Childs Nerv Syst 2010; 26 (10): 1417-33. Epub 2010 Aug 10.

3.- López R, López JC, Belendez C, et al: Tumores vasculares en la infancia. An Pediatr (Barc) 2009; 72 (02): 143. e1-e15.

4.- O-Lee TJ, Messner A: Subglottic Hemangioma. Otolaryngol Clin N Am 2008; 41: 903-11.

5.- Leaute-Labreze C, Dumas de la Roque E, Hubiche T, et al: Propranolol for severe hemangiomas of infancy. N Engl J Med 2008; 358: 2649-51.

6.- Denoyelle F, Leboulanger N, Enjolras O, et al: Role of propranolol in the therapeutic strategy of infantile laryngotracheal hemangioma. Int J Pediatr Otorhinolaryngol 2009; 73: 1168-72.

7.- Buckmiller L, Dyamenahalli U, Richter GT: Propranolol for airway hemangiomas: case report of novel treatment, Laryngoscope 2009; 119: 2051-4.

8.- Sebastian B, Kleinsasser O: Zur Behandlung der Kehlkopfhämangiome bei Kindern, Laryngol. Rhinol Otolaryngol 1984; 63: 403-7.

9.- Hoeve LJ, Kuppers G, Verwoerd C: Management of infantile subglottic hemangioma: laser vaporization, submucous resection, intubation or intralesional steroids? Int J Pediatr Otorhinolaryngol 1997; 42: 179-86.

10.- Cotton RT, Tewfik T: Laryngeal stenosis following carbon dioxide laser in subglottic hemangioma. Ann Otol Rhinol Laryngol. 1985; 94: 494-7.

11.- Vijayasekaran S, White D, Hartley BEJ, et al: Open excision of subglottic hemangiomas to avoid tracheostomy. Arch Otolaryngol Head Neck Surg 2006; 132: 159-63.

12.- Bajaj Y, Hartley BJ, Wyatt ME, et al: Subglottic haemangioma in children: experience with open surgical excision. J Laryngol Otol 2006; 120: 1033-7.

13.- Jephson C, Manunza F, Syed S, et al: Successful treatment of isolated subglottic haemangioma with propranolol alone. Int. J. Pediatr Otorhinolaryngol 2009; 73: 1821-3.

14.- Maturo $S$, Hartnick $C$ : Initial experience using propranolol as the sole treatment for infantile airway hemangiomas. Int J Pediatr Otorhinolaryngol 2010; 74: 323-5.

15.- Leboulanger N, Fayoux P, Teissier N, Cox A, et al: Propranolol in the therapeutic strategy of infantile laryngotracheal hemangioma: A preliminary retrospective study of French experience. Int J Pediatr Otorhinolaryngol 2010; 74: 1254-7.

16.- Truong M, Perkins J, Messner A, et al: Propranolol for the treatment of airway hemangiomas: A case series 
and treatment algorithm. Int J Pediatr Otorhinolaryngol 2010; 74: 1043-8.

17.- Storch $\mathrm{CH}$, Hoeger PH: Propranolol for infantile haemangiomas: insights into the molecular mechanisms of action. B J Dermatol 2010; 163: 269-74.

18.- Peridis S, Pilgrim G, Athanasopoulos I, Parpounas K: A meta-analysis on the effectiveness of propranolol for the treatment of infantile airway haemangiomas. Int $\mathrm{J}$ Pediatr Otorhinolaryngol 2011; 75 (4): 455-60. Epub 2011 Feb 17.

19.- Menezes M, McCarter R, Greene E, et al: Status of Propranolol for Treatment of Infantile Hemangioma and Description of a Randomized Clinical Trial. Ann Otol Rhinol Laryngol 2011; 120 (10): 686-95.

20.- Cushing SL, Boucek RJ, Manning SC, et al: Initial experience with a multidisciplinary strategy for initiation of propranolol therapy for infantile hemangiomas. Otolaryngol Head Neck Surg 2011; 144 (1): 78-4.
21.- Canadas K, Baum E, Lee $S$, et al: Case report: Treatment failure using propranolol for treatment of focal subglottic hemangioma. Int J Pediatr Otorhinolaryngol 2010; 74: 956.8.

22.- Javia L, Zur K, Jacobs I: Evolving treatments in the management of laryngotracheal hemangiomas: Will propranolol supplant steroids and surgery?. Int J Pediatr Otorhinolaryngol 2011; 75: 1450-4.

23.- Goswamy J, Rothera M, Bruce I: Failure of propranolol in the treatment of childhood haemangiomas of the head and neck. J Laryngol \& Otol 2011; 125: 1164-2.

24.- Raol N, Metry D, Edmonds J, et al: Propranolol for the treatment of subglottic hemangiomas. Int J Pediatr Otorhinolaryngol 2011; 75: 1510-4.

25.- Fuchsmann C, Quintal M, Giguere C, Ayari-Khalfallah $S$, et al: Propranolol as First-line Treatment of Head and Neck Hemangiomas. Arch Otolaryngol Head Neck Surg 2011; 137 (5): 471-8. 